\title{
Aerial, GIS, and Field Data Calibration of a Landsat ETM+ Mountain Pine Beetle Detection Procedure in British Columbia, Canada
}

\author{
R. Skakun^, S. E. Franklin^^, M. A. Wulder , A. Carroll \\ ${ }^{\wedge}$ University of Calgary, Department of Geography, Calgary, Alberta T2N 1N4 \\ Canadian Forest Service, Pacific Forestry Centre, Victoria, British Columbia V8Z 1M5
}

\begin{abstract}
The identification and classification of mountain pine beetle, Dentroctonus ponderosa (Hopkins), red-attack damage patterns in a mature lodgepole pine Pinus contorta) forest located in the Fort St. James Forest District, British Columbia, was accomplished using 1999 Landsat ETM+ satellite imagery, a 1999 mountain pine beetle field and aerial survey point dataset, and GIS forest inventory data. A maximum likelihood classification based on training areas developed from stratified field and aerial survey points was $73 \%$ correct. The red-attack damage class showed forest stands with a high likelihood of containing small groups of red-attacked trees.
\end{abstract}

\section{INTRODUCTION}

In many areas of the west-central Interior region of British Columbia, populations of mountain pine beetle have reached epidemic proportions. The implications of a large outbreak include widespread damage, usually expressed as the mortality of hundreds of thousands of lodgepole pine trees. The mountain pine beetle attacks may create a previsual signal, sometimes known as the green-attack stage, when changes to cellular structures (perhaps caused by water deficiency) precede visually-obvious degradation of foliage (Murtha, 1978). Visually, damage is often first apparent as red-attack colouration, which usually appears shortly after the initial attack (Figure 1). Red-attack may be followed by a grey-attack stage which typically indicates high mortality (Unger, 1993).

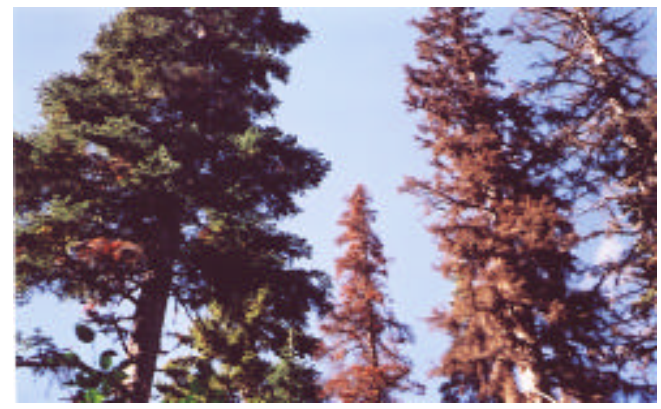

Figure 1. Red-attack defoliation. (Photographed by Rob Skakun, Fort St. James area, September 4, 2001).

Research to detect and classify red-attack damage and forests severely infested by mountain pine beetles using remotely-sensed data, acquired from sensors such as the
Landsat Thematic Mapper (Renz and Nemeth, 1985) and by aerial multispectral sensors (Gimbarzevsky et al., 1992), has been well documented, but few operational examples exist. The main problems have been twofold: 1) there is a very high degree of natural variability in forests affected by the beetle; and, 2) there is a relatively small influence of beetle damage on spectral response, particularly as measured by satellite sensors, unless very large and homogeneous areas are affected. This research focused on a judicious use of the available imagery with field, aerial survey, and GIS data to classify red-attack damage caused by mountain pine beetle infestation.

\section{STUDY AREA AND DATA COLLECTION}

The $5070 \mathrm{~km}^{2}$ study area is located in the lower southern half of the Fort St. James Forest District and extends into the northeast and northwest corners of the Vanderhoof and Prince George Forest Districts in British Columbia (Figure 2).

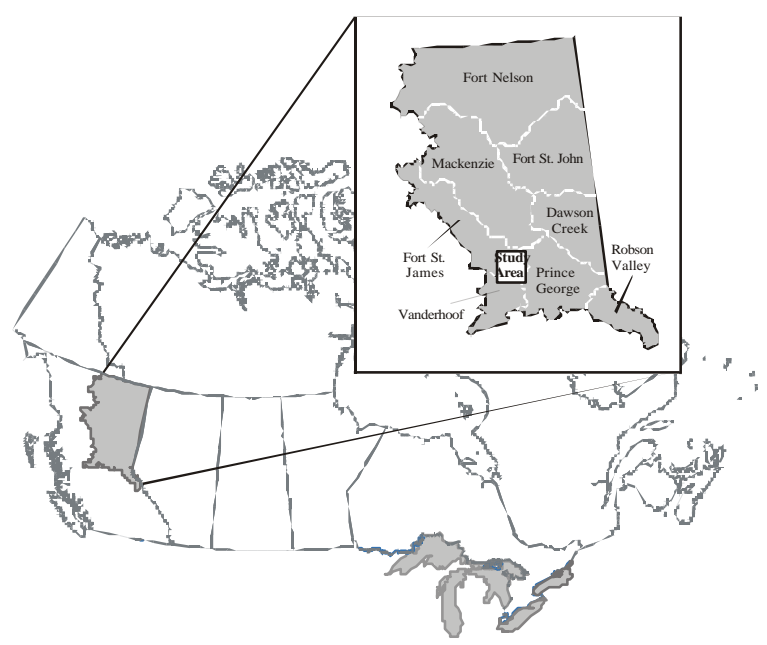

Figure 2. Location of study area in British Columbia.

The main forest species in the study area include: lodgepole pine, white spruce (Picea glauca), Douglas-fir (Pseudotsuga menziessi), balsam fir (Abies balsamea), and trembling aspen (Populus tremuloides). Of these forest species, lodgepole pine stands are typically the youngest and the most dense in terms of canopy closure. The lodgepole pine stands cover approximately $61 \%$ of the total forested area. According to the British Columbia 
forest inventory classification, the lodgepole pine stands average age class is 6 (101-120 years), average height class is 3 (19.5-28.4 metres), and average canopy closure class is $5(46-55 \%)$.

The mountain pine beetle field and aerial survey point dataset was collected from August 9 to September 10, 1999. An observer in a helicopter counted the number of red-attack trees directly below the aircraft, at a flying altitude of 100-200 feet, and recorded the location into one of three categories: <10, 10-20, and 21-50 trees (M. Paulson, TerraPro GPS Surveys Ltd., pers. comm.). For example, a point collected in the $<10$ trees category represents an epicentre containing less than 10 red-attack trees in an approximate 50 metre diameter plot area. A ground crew then walked the area around a sample of these sites and recorded the following field data:

- type of attacking beetle(s) (up to four)

- possible fire and water damage at the site

- location near road, cut-block edge, swamp, creek, lake, or beside salvage and poor site conditions

- $\quad$ presence of faders

- $\quad$ single and scattered red-attack and grey-attack defoliation (number of trees)

In total, 2249 mountain pine beetle field and aerial survey points were recorded in the study area. Most of the observations were in the $<10$ red-attacked tree category, which comprises $82 \%$ of the total mountain pine beetle field and aerial survey points.

The satellite data consisted of a Landsat 7 ETM+ image (Path/Row: 49/22) acquired on Sept 12, 1999 with 0\% cloud cover. The image solar conditions were $38.6^{\circ}$ elevation and $160.3^{\circ}$ azimuth. The image was atmospherically corrected using a standard atmospheric model (Richter, 1990) and georeferenced to a UTM NAD 83 Zone 10 projection.

A GIS forest inventory polygon dataset was used to develop strata from the structural components of the forest in the study area. Each of the forest polygons contained a large number of attribute data including: species composition, stand age (years), canopy closure (\%), height $(\mathrm{m})$, dbh (diameter breast height, $\mathrm{cm})$, volume $\left(\mathrm{m}^{3}\right)$, and area (ha). The dataset was structured in vector format, projected at a 1:250,000 scale, and referenced over NTS Map Sheet Numbers 93J and 93K. The geographical coordinates were in metres, projected to UTM Zone 10 with a NAD 83 datum.

\section{METHODS}

A supervised classification approach was adopted based on the large number of possible training area pixels contained in the field and aerial survey dataset. First, a mountain pine beetle susceptibility mask was generated based on a set of host susceptibility factors; for example, Unger (1993) suggested that stand susceptibility to mountain pine beetles increase "with trees over 60 years of age and stands with a high pine component." We removed from further consideration all field sample points which were located in polygons composed of $<40 \%$ lodgepole pine or which were <60 years of age; of the polygons remaining, 93\% were composed of lodgepole pine as the dominant species covering an area approximately 19,100 ha. Some spruce beetle and western balsam bark beetle defoliation occurred and, where noted in the field sample, were deleted. A similar screening decision-rule was applied to remove grey-attack points from the sample; and finally, an edge filter was used to delete points located near and on the edges of cut-blocks, roads, rivers, and lakes.

The resulting mountain pine beetle red-attack training area was comprised of 360 pixels from only those areas observed in the field or the aerial survey which were likely mountain pine beetle red-attack damage at the time of the data collection. We acquired a similar size training area for non-attacked forest by sampling from the large number of polygons in the GIS which did not contain beetle damage but which satisfied the stratification criteria. We then divided the training pixels into two equal samples, and reserved one of these to test the accuracy, using the other to generate class signatures for input to the maximum likelihood procedure.

\section{RESULTS AND DISCUSSION}

The classification accuracy assessment revealed that overall the non-attack and red-attack damage classes were $72.3 \%$ correct (Table 1). The red-attack defoliation class was $73.3 \%$ correct based on the spectral response data obtained following stratification of the mountain pine beetle field and aerial calibration points. The non-attack accuracy of $71.1 \%$ is reasonable; this level of accuracy is consistent with forest classification results reported in a wide of range of relatively homogeneous conifer stands and simple maximum likelihood decision rules (e.g., Franklin and Luther, 1995).

Table 1. Accuracy assessment of supervised classification.

\begin{tabular}{lccc} 
& \multicolumn{3}{c}{ Percent of pixels classified into a class } \\
\cline { 2 - 4 } Non-attacked & Red-attacked & Total \\
\cline { 2 - 4 } Non-attacked & 71.1 & 28.9 & 100 \\
Red-attacked & 26.7 & 73.3 & 100 \\
\hline
\end{tabular}

Mean classification accuracy: 72.3

The map of red-attacked forest stands is shown in Figure 3. Two small zoom in windows indicate the patchy nature of the red-attack damage class within stand polygons. Each ETM+ pixel in the red-attack class represents at least 10 red-attack trees in that small area; polygons with several 
small patches represent stands in which beetles were relatively abundant. Some relatively large homogeneous patches indicate high infestation areas. The general appearance, however, is one of reasonableness; the degree of detection and classification is consistent with the way in which mountain pine beetle infestations occurred in this area. The maps appear useful in decision-making for managers.

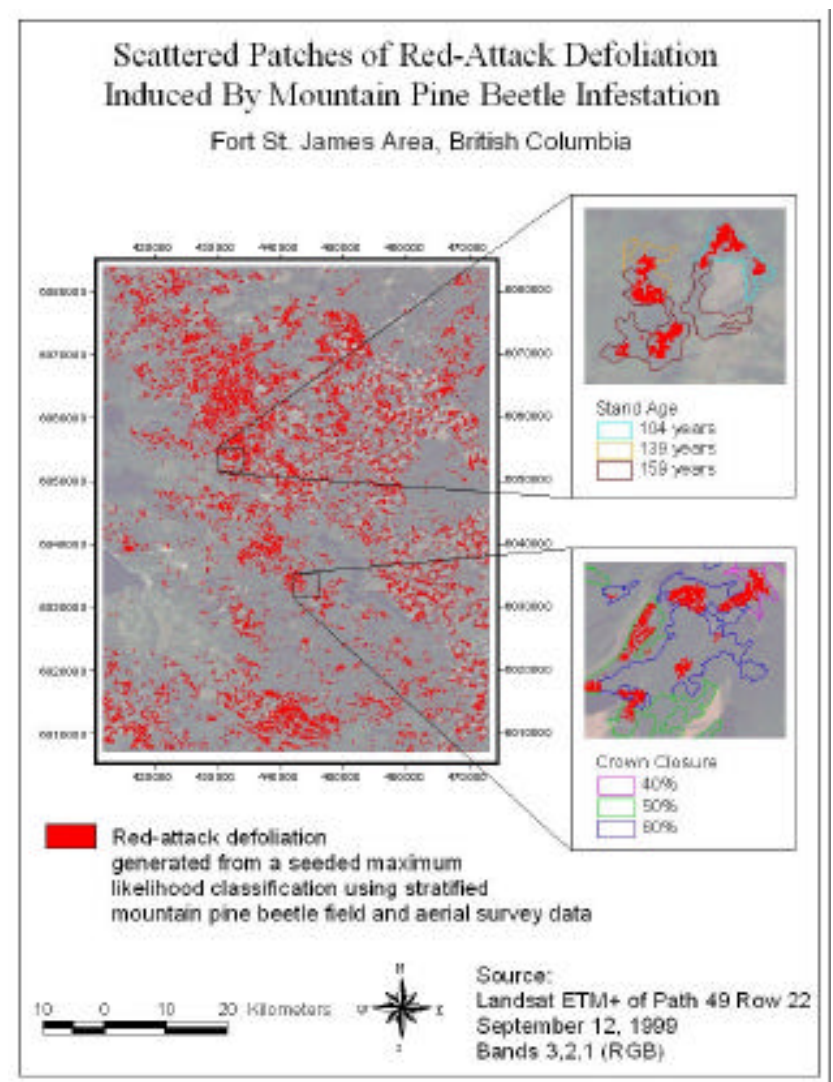

Figure 3. Classification of mountain pine beetle red-attack damage class in the Fort St. James area.

Stratification of a large number of field and aerial survey points resulted in a distinct spectral signature for red-attack damage; damage caused by the mountain pine beetle was not confounded by uncontrolled natural stand variability and the relatively small spectral influence of a few damaged crowns within a small area. This technique has been used to improve classification results of spruce budworm defoliation in conifer stands (Franklin and Raske, 1994); for example, $75 \%$ overall accuracy was achieved in western Oregon based on the stratification of a Landsat TM image acquired prior to the beetle infestation (Franklin et al., 1995).

\section{CONCLUSION}

Red-attack damage attributable to mountain pine beetle infestation in the Fort St. James Forest District was classified with Landsat ETM+ imagery using training areas developed from a mountain pine beetle field and aerial survey point dataset. To reduce training area variability, we stratified the training areas prior to signature generation with GIS data and logical decision rules based on host susceptibility information and forest structure. This was important because the high variance in spectral response within training areas can cause an over-estimation of the red-attack damage class during supervised classification procedures. The maximum likelihood classification accuracy was determined to be approximately $73 \%$, based on 360 independent mountain pine beetle field and aerial survey validation points. The final classification map showed small pockets of infestation - individual pixels within forest stands - which were likely the locations of mountain pine beetle red-attack damage.

\section{ACKNOWLEDGEMENTS}

This work was funded by a research grant from the Natural Sciences and Engineering Research Council and a research contract from the Canadian Forest Service.

\section{REFERENCES}

[1] Franklin, S. E., and J. E. Luther. 1995. Satellite remote sensing of balsam fir forest structure, growth, and cumulative defoliation. Can. J. Rem. Sensing, 21: 400-411. [2] Franklin, S.E., and A. Raske. 1994. Satellite remote sensing of spruce budworm defoliation in western Newfoundland. Can. J. Rem. Sensing, 20: 37-48.

[3] Franklin, S.E., R.H. Waring, R.W. McCreight, W.B. Cohen, and M. Fiorella. 1995. Aerial and satellite sensor detection and classification of western spruce budworm defoliation in a subalpine forest. Can. J. Rem. Sensing, 21: 299-308.

[4] Gimbarzevsky P., A.F. Dawson, and G.A. Van Sickle. 1992. Assessment of aerial photographs and multispectral scanner imagery for measuring mountain pine beetle damage. For. Can., Pac. and Yukon region. Info. Rep. BC-X-333.

[5] Murtha, P.A. 1978. Remote sensing and vegetation damage: a theory for detection and assessment. Photogramm. Eng. Rem. Sensing, 44: 1147-1158.

[6] Renz, A.N. and J. Nemeth. 1985. Detection of mountain pine beetle infestation using Landsat MSS and simulated Thematic Mapper data. Can. J. Rem. Sensing, 11:50-57.

[7] Richter, R. 1990. A fast atmospheric correction algorithm applied to Landsat TM Images. Int. J. Rem.

Sensing, 11: 159-166.

[8] Unger, L. 1993. Mountain pine beetle. Forestry Canada, Forest Insect and Disease Survey, Forest Pest Leaflet No. 76, 7 p. 